\title{
Dynamics of an IPM pest-predator model with impulses and stage structure on predator population
}

\author{
Jianjun Jiao ${ }^{1}$, Shaohong Cai ${ }^{1}$, and Qi Quan ${ }^{1}$ \\ ${ }^{1}$ Guizhou University of Finance and Economics
}

May 8, 2020

\begin{abstract}
Integrated pest management(IPM) has been promoted as an environmentally friendly pest control approach. It utilizes a combination of control methods to control pest populations in agricultural and forestry systems. In this paper, we propose an IPM pest-predator model with impulses and stage structure on predator population, where the predator population is divided into two stages, a juvenile stage and a mature stage. The mature predator's predation conversion for production of new predators. This kind of stage-structured pest-predator model has been omitted in the mathematical models for integrated pest management. The dynamical properties for the pest-extinction solution and permanence of system $\$(2.1) \$$ are established. The simulations are employed to support the proofs. Our results provide a good balance between the biological control and chemical control for integrated pest management.
\end{abstract}

\section{Hosted file}

Jiao, Cai and Quan.pdf available at https://authorea.com/users/319683/articles/449394dynamics-of-an-ipm-pest-predator-model-with-impulses-and-stage-structure-on-predatorpopulation 
figures/1/1-eps-converted-to.pdf 
figures/2/2-eps-converted-to.pdf 
figures/3/3-eps-converted-to.pdf 
figures/4/4-eps-converted-to.pdf 
figures/5/5-eps-converted-to.pdf 
figures/6/6-eps-converted-to.pdf 
figures/7/7-eps-converted-to.pdf 
figures/8/8-eps-converted-to.pdf 
figures/9/9-eps-converted-to.pdf 
figures/10/10-eps-converted-to.pdf 
figures/11/11-eps-converted-to.pdf 
figures/12/12-eps-converted-to.pdf 